pigmentosa, and, moreover, on pharmacological grounds it would be unlikely for aspirin, an acid substance, to have such an action, as most recognized histamine liberators are bases.

Another possibility would be that aspirin was acting as a hapten, forming an antigen when combined with serum proteins. We have no clear evidence against this, but we would have to assume that at least one in five patients who suffered from chronic urticaria would also have such an allergy to aspirin.

That aspirin in some way renders the skin in these patients more sensitive to histamine is suggested by the present report that intradermal histamine weals were larger after aspirin in these patients. In this connexion it is interesting that Juhlin and Rune (1962) have reported that the threshold to $48 / 80$, a known histamine liberator, is lowered in acute urticaria.

It has been stated in the past that aspirin sensitivity is specific to acetylsalicylic acid and does not extend to related compounds. In the present investigations similar reactions were obtained in most patients from sodium salicylate and phenyl salicylate, and it would seem likely that it is the salicylic acid radical which is responsible for aspirin reactivity.

Whatever the underlying cause, it is now established that a point of great practical importance in the management of patients with urticaria is to avoid any administration of salicylates.

The investigation of a patient with chronic urticaria, aspirin reactivity, and jaundice revealed abnormal liver histology described as atypical portal cirrhosis. This finding prompted the investigation of all patients who reacted to aspirin. To date, two other similar cases have been found. It is intended to report later the details of these three cases with the triad of chronic urticaria, aspirin reactivity, and liver disease.

\section{Summary}

Aspirin-containing drugs will cause exacerbation in some patients with chronic urticaria. In 112 out of 228 patients with chronic urticaria such a reaction could not be excluded from the history, and provocative test doses of aspirin were given ; 50 patients (22\%) developed a well-marked increase in the urticaria. No clinical differences could be observed between the patients who reacted in this way and those who did not.

This action of aspirin is probably due to the salicylic acid radical, as most patients tested have had similar reactions with doses of sodium salicylate and phenyl salicylate.

It has been shown in eight patients that the increase in wealing is proportional to the dose of aspirin administered. In four patients who did not react at all to $300 \mathrm{mg}$. of aspirin there were successively more severe reactions to 600 and 1,200 mg. In four others the reaction increased so much between 300 and $600 \mathrm{mg}$. that a larger dose was not given. The quantitative effect is further supported by the clinical observation that some patients have developed exacerbations when large doses of aspirin have been taken and have shown no reaction to isolated small doses.

With appropriate measurements, before and after aspirin, it has been shown that there was no effect on the physical urticarias, including 10 patients with dermographism, five with exertion urticaria (cholinergic), and three with cold urticaria. Similarly, no effect was demonstrated in the wealing in four patients with urticaria pigmentosa.

It has been demonstrated that induced intradermal histamine weals measured four hours after $600 \mathrm{mg}$. of aspirin were larger in a group of patients who had reacted to aspirin than in a control group. It is suggested that the mode of action of aspirin in chronic urticaria is by enhancing the effect of histamine in the skin.

This work was carried out with the help of an M.R.C. grant, and we should like to express our thanks for this. We also wish to thank Dr. C. D. Evans, Dr. R. R. M. Harman, and other colleagues who have so kindly referred cases to us. We are grateful to Mr. F. Wellington, Chief Pharmacist, Bristol General Hospital, who gave us invaluable help.

\section{REFERENCES}

Calnan, C. D. (1957). Lancet, 1, 996.

Hamrin, B. (1957). Ibid., 1, 867.

Juhlin, L., and Rune, C. (1962). Acta derm.-venereol. (Stockh.), 42, 11 Warin, R. P. (1960). Brit. F. Derm., 72, 350.

\title{
Clinical Measurement of the Anti-inflammatory Effects of Salicylates in Rheumatoid Arthritis
}

\author{
P. L. BOARDMAN,* M.D., M.R.C.P. ; F. DUDLEY HART,* M.D., F.R.C.P.
}

Salicylates in relatively low dosage modify certain components of the inflammatory reaction in animal experiments (Adams and Cobb, 1963). Theoretically they may be used in rheumatoid arthritis to promote the relief of pain or for their antiinflammatory effect. It is important to determine whether there is a different dose-response relationship for each of these characteristics.

The cardinal signs of inflammation-pain, heat, redness, and swelling-are the indices available for the direct clinical assessment of inflammatory activity in rheumatoid arthritis. Pain is subjective and difficult to quantitate. Joint heat was studied by Smyth and Clark (1957) and by Vaughn, Howell, and Kiem (1959); it was found to be of little value. The latter authors also found redness an unsatisfactory index for assessment. Hart and Clark (1951) introduced the method of standard

* Westminster, Hospital, London S.W.1. jewellers' rings for the measurement of joint size, by which accurate serial recordings could be made.

Fremont-Smith and Bayles (1965) investigated the effect of high dosage of salicylate in 11 patients in hospital with rheumatoid arthritis. Aspirin was given orally in increasing quantity over a five-day period until the largest tolerated dose was reached-on average, $5.2 \mathrm{~g} . / 24$ hours. In most cases dosage increase was stopped because of tinnitus or deafness. Potent analgesics without anti-inflammatory action were then abruptly substituted. An increase in joint size, measured by standard jewellers' rings, and deterioration of grip strength, symptoms, and other indices followed rapidly the withdrawal of aspirin. They concluded that all patients with active rheumatoid arthritis, whether it was mild or severe, should receive salicylates regularly in the largest tolerated dosage in the absence of obvious contraindications. Because of sideeffects "blind" techniques and quantitation of the changes were not attempted. 
A further point requiring critical consideration is whether the rebound deterioration following cessation of treatment is the direct consequence of the withdrawal of an anti-inflammatory agent or whether more complex mechanisms are of significance (Bywaters, 1963).

The aim of this study was to establish a quantitative method for measuring joint size by a technique based on the use of standard jewellers' rings under double-blind controlled conditions, and to differentnate reduction of swelling, an index of anti-inflammatory activity, from pain relief or analgesic effect with various doses of salicylates.

The results of the following four trials are reported: (1) comparison of prednisone with placebo to determine the effect of a known anti-inflammatory agent ; (2) comparison of paracetamol with placebo to determine the effect of a known analgesic agent; (3) comparison of high dosage of salicylate with low dosage ; and (4) comparison of low dosage of salicylate with placebo.

\section{Material and Methods}

Each trial was in the form of a double-blind cross-over comparison. The results were expressed sequentially, preference being based on alteration of joint size during the two periods.

All patients had classical or definite rheumatoid arthritis (American Rheumatism Association, 1959) of at least one year's duration. They were selected from consecutive attenders at the rheumatism clinic provided that they had synovitis of the small joints of the hands and that there was no history of intolerance to the relevant drugs. Only those patients were included in whom it was possible to stop all treatment for 14 days before the trial.

In trial 1 prednisone $7.5 \mathrm{mg}$. daily was compared with placebo, both being administered as a single evening dose. In trial 2 paracetamol $6 \mathrm{~g}$. daily was compared with placebo ; each was administered as three tablets four times daily. In trial 3 high dosage of salicylate was given at $5.3 \mathrm{~g}$. (80 gr.) daily in four equal doses and low dosage at $2.6 \mathrm{~g}$. (40 gr.) daily. In trial 4 low dosage of salicylate, $2.6 \mathrm{~g}$. daily, was compared with placebo.

Each drug was given for seven consecutive days, each trial lasting 14 days. All tablets were taken after food. Salicylate was given in its glycinated form as a single tablet containing $660 \mathrm{mg}$. (10 gr.). After each treatment period the bottles were collected and the number of tablets remaining was checked.

Patients were interviewed a minimum of four times at weekly intervals in each trial. At the first visit joint size and grip strength were measured. These indices were measured again at the second visit. If the difference in joint size was less than $4 \mathrm{~mm}$. and the overall change of grip strength less than $50 \mathrm{~mm}$. $\mathrm{Hg}$ they were regarded as having a stable baseline and the trial was started. In those with greater changes further baseline assessment was made until the indices became stable. At the third visit-the end of the first seven-day trial periodthe indices were measured and side-effects recorded, direct questions being avoided. The same programme was followed at the fourth visit, which marked the end of the trial, and the individual preference for either of the treatment periods was noted.

Joint size was measured as the circumference of the proximal interphalangeal joints of the four fingers of each hand, and the distal interphalangeal joint of each thumb-that is, the sum of the circumference measurements of the 10 digits. It was measured by a gauge (Fig. 1), the actual readings being recorded to the nearest millimetre. This gauge was used instead of standard rings, as it is quicker, the result is less likely to be influenced by the frictional state of the skin, and the tension at which it is applied is probably more consistent, particularly over a tender joint. It is also preferred by the patient. The possibility of error developing at the extreme ranges of the scale because of variation in tension of the plastic spring was considered, but comparison against the rigid standard rings excluded significant inaccuracy. In Fig. 2 the results of 100 pairs of measurements of joint size with the two instruments are plotted. There is good correlation throughout the whole range.

Grip strength was measured for each hand with the cuff inflated to $30 \mathrm{~mm}$. $\mathrm{Hg}$, the result of a single sustained squeeze being recorded.

Joint size was measured before grip strength, or preference was assessed to avoid bias from knowledge of the other indices. The measurements were recorded without knowledge of those obtained at the previous visit.

One observer (P. L. B.) carried out all assessments. Variation between observers may be responsible for differences greater than significant changes in joint size. Table I gives the results obtained by observers in practice and those without previous experience of the method. Each of three observers with previous practice measured the joint size of 10 patients with rheumatoid

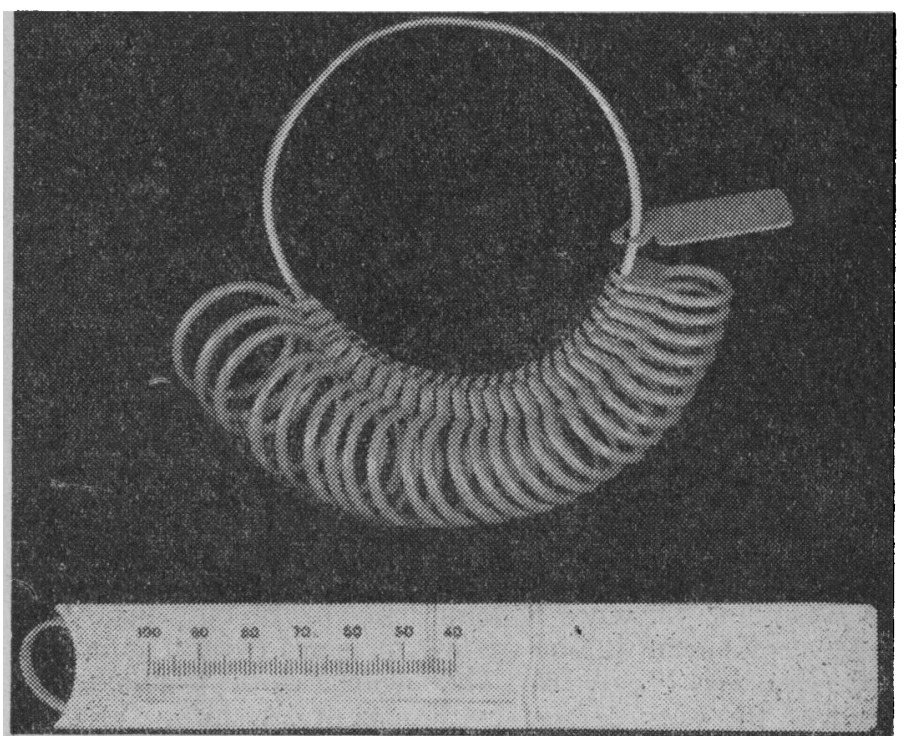

FIG. 1.-Photograph of gauge for measuring joint size in millimetres; standard jeweller's rings are also shown.

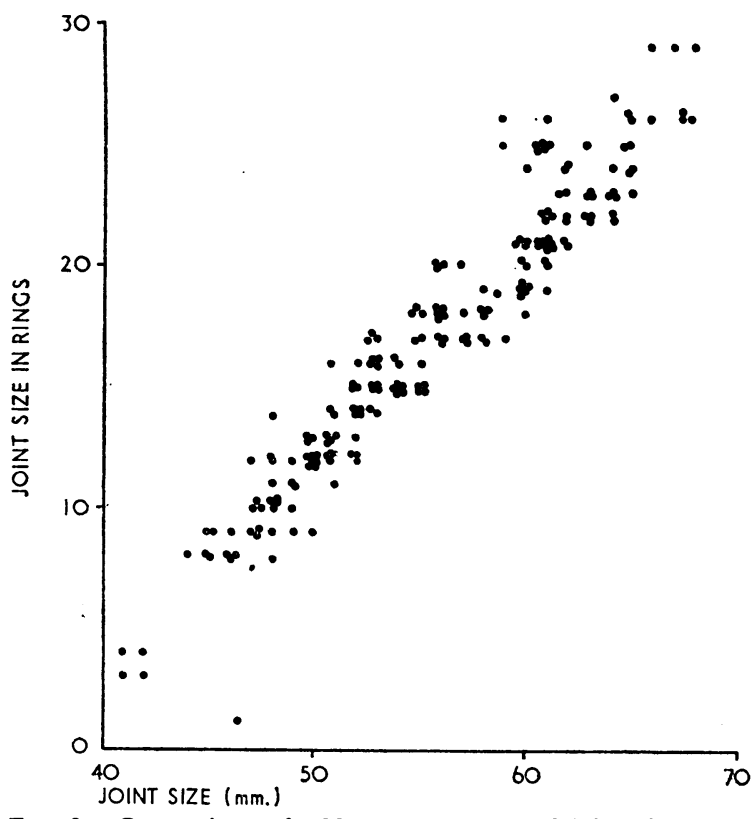

FIG. 2.-Comparison of 100 measurements of joint size made on the gauge with those obtained with standard rings. Coefficient correlation 0.948 . 
arthritis twice at an interval of an hour. The average difference between the two readings was $3.0 \mathrm{~mm}$. For three observers without previous experience of the method, under similar conditions, the mean difference was $4.5 \mathrm{~mm}$.

All patients were seen at the same time of day. In a group of eight patients in hospital with active rheumatoid arthritis there was an apparent decrease of joint size throughout the day (Fig. 3). The grip strength was greater in the evening than in the morning, the usual diurnal pattern (Wright, 1959), but the difference was not statistically significant.

TABL. I.-Observer Variation. Comparison of Results Obtained by Three Observers with Previous Experience of the Method with Similar Readings made by Three Without Prior Practice

\begin{tabular}{c|c|c}
\hline & Difference between Measurements (mm.) \\
\hline Observer & With Practice & Without Practice \\
\hline 1 & 3.0 & 3.8 \\
2 & 2.9 & 5.1 \\
3 & 3.2 & 4.6 \\
\hline Mean & 3.0 & 4.5 \\
\hline
\end{tabular}

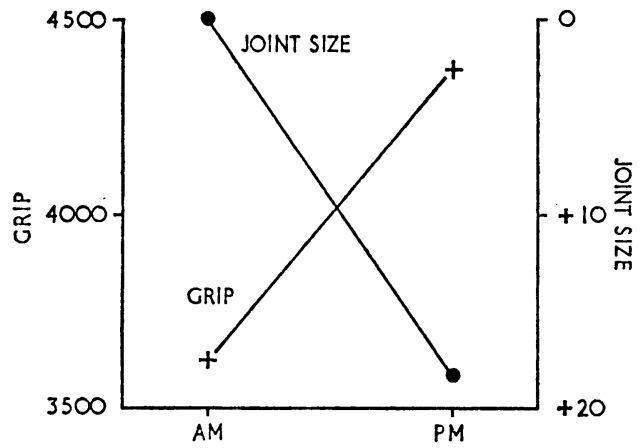

FIG. 3.-Diurnal variation of joint size and grip strength in eight patients with rheumatoid arthritis. Joint size is measured in millimetres (t Indicates improvement). Grip strength is measured in mm. mercury, the sum for each rime being plotted.

To express the results sequentially it is necessary to define a significant difference of joint size. In 50 outpatients with rheumatoid arthritis joint size was measured twice at an interval of seven days (Table II). The readings at the beginning and end of the week differed by $3 \mathrm{~mm}$. or less in $42(84 \%)$ of the patients. The mean difference was $2.12 \mathrm{~mm}$. (S.D. 1.4). In eight patients $(16 \%)$ the difference was $4 \mathrm{~mm}$. or greater. A difference of $4 \mathrm{~mm}$. was therefore arbitrarily assumed to be significant over a seven-day period.

TABLB II.-Significant Difference in Joint Size

\begin{tabular}{lll|l|l|l|l|l|l|l}
\hline $\begin{array}{l}\text { Difference in joint size (mm.) } \\
\text { No. of patients }\end{array}$ &. &. & & 0 & 1 & 2 & 3 & 4 & 5 \\
\hline
\end{tabular}

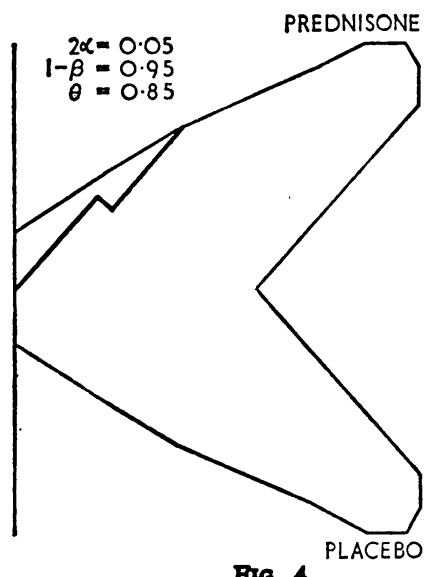

Fio. 4

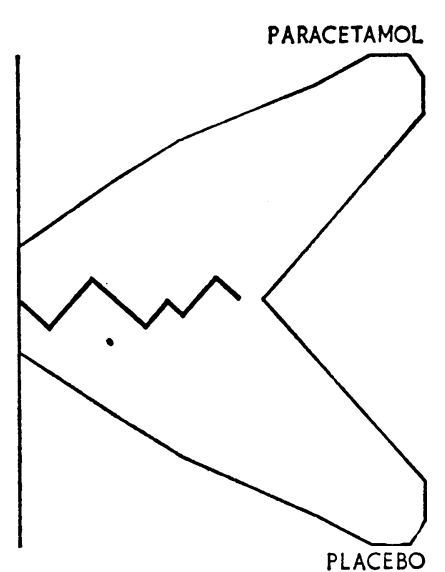

Fro. 5
PLACEBO

\section{Results}

Trial 1: Prednisone v. Placebo.-A significant decrease of joint size on prednisone compared with placebo was reached after plotting 11 results (Fig. 4). As there was a difference of less than $4 \mathrm{~mm}$. of joint size in two patients, the total number involved in the trial was 13 . There was a significant increase in grip strength on prednisone compared with placebo in those patients in whom there was reduction of swelling. The mean grip strength on prednisone was $379 \mathrm{~mm}$. $\mathrm{Hg}$ and on placebo $292 \mathrm{~mm}$. $\mathrm{Hg}(\mathrm{t}=2.3 ; \mathrm{n}=20 ; 0.05>\mathrm{P}>0.02)$. The preference was for prednisone in 8 of the 11 patients, 1 preferred placebo, and 2 found no difference.

Trial 2 : Paracetamol o. Placebo.-There was no significant difference of joint size in patients on paracetamol compared with placebo (Fig. 5). Of the 14 fulfilling the criteria for inclusion in the sequential analysis seven had less swelling on paracetamol and seven on placebo. Four patients were excluded from this trial because they had inadequate alteration of joint size. The 14 patients included in the trial had a mean improvement of grip strength from $303 \mathrm{~mm}$. $\mathrm{Hg}$ on placebo to $326 \mathrm{~mm}$. $\mathrm{Hg}$ on paracetamol. This difference is not significant $(t=0.57 ; n=26 ; P>0.05)$. The preference was for paracetamol in six and for placebo in three patients, five finding them to be equally effective.

Trial 3: High Dosage of Salicylate o. Low Dosage.-There was a significant decrease of joint size on high dosage of salicylate compared with low dosage after 14 results were plotted (Fig. 6). In four patients joint size did not change sufficiently for inclusion in the analysis. The mean grip strength was $322 \mathrm{~mm}$. $\mathrm{Hg}$ on high dosage compared with $309 \mathrm{~mm}$. $\mathrm{Hg}$ on low dosage. This difference is not significant $(t=0.3 ; n=26$; $P>0.05$ ). The high-dosage regimen was preferred by 11 patients and the low dosage by one, two noting no difference.

Trial 4: Low Dosage of Salicylate v. Placebo.-There was no significant difference in joint size on low dosage compared with placebo (Fig. 7) after 15 results were plotted. In nine patients joint size did not change significantly on either preparation: these were discarded from the analysis. The mean grip strength on low dosage of salicylate was $312 \mathrm{~mm}$. $\mathrm{Hg}$ compared with $348 \mathrm{~mm}$. Hg on placebo. In five patients the preference was for low dosage and in three for placebo, seven finding no difference.

\section{Side-effects}

In trial 1 two out of 13 patients had subjective reactions on prednisone.

In trial 2 side-effects occurred in 10 out of 18 on paracetamol - generally dyspepsia and drowsiness-compared with three on placebo, all of which were dyspepsia.

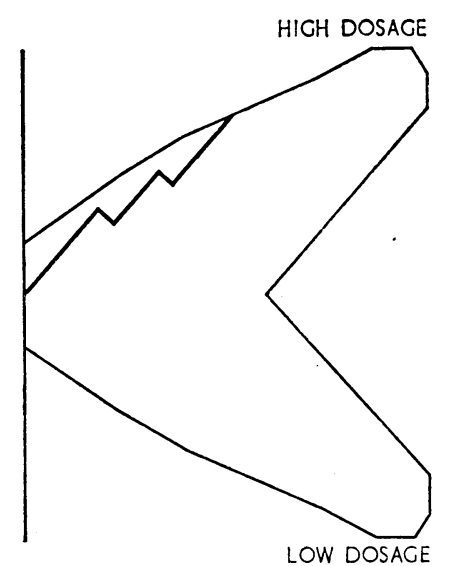

Fia. 6

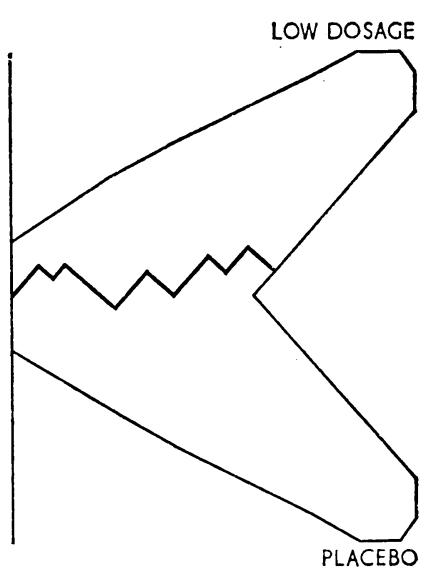

Fig. 7
Fig. 4.-Comparison of ioint size on prednisone with placebo. Fig. 5-Comparison of joint size on paracetamol with placebo. Fig. 6.-Comparison of high dosage of salicylate with low dosage. Fig. 7.-Comparison of low dosage of salicylate with placebo. 
High dosage of salicylate (trial 3) was associated with sideeffects in six out of 18 patients, the complaint being tinnitus in four and nausea in two. On low dosage giddiness and abdominal distension were each noted once.

In trial 4 four out of 15 had complaints on low dosage of salicylate. These were frequency of micturition (1), giddiness (1), and dyspepsia (2). Placebo was associated with side-effects in three patients, two complaining of depression and one of a hot feeling.

\section{Discussion}

Trial 1 illustrates the effect of a known anti-inflammatory agent. Significant reduction of joint swelling is associated with improvement of grip strength and preference for prednisone.

Trial 2 defines the pattern that is obtained from a known analgesic agent without anti-inflammatory action: There is no difference of joint size on paracetamol compared with placebo. Both grip strength and preference suggest that there is some benefit from paracetamol compared with placebo, though neither alters to a statistically significant extent.

These findings suggest that under certain conditions alteration of joint size may be related specifically to reduction of inflammation. Grip strength and preference are less specific indices in that they may also improve after the administration of analgesic agents.

In trial 3 high dosage of salicylate is associated with reduction of joint size when compared with low dosage. The preference is strongly in favour of high dosage. The mean grip strength is better on high dosage, though the change does not reach significant levels.

There is no difference of joint size on low dosage of salicylate compared with placebo, a point shown in trial 4 . There is no difference of preference: the grip strength is better on placebo but not to a significant extent.

A possible disadvantage of sequential analysis is the introduction of selection, because experiments in which there is no significant difference are discarded. In our study this is accepted deliberately to exclude the patient who lacks reversible soft-tissue inflammatory swelling. The alteration of joint size that follows the administration of an anti-inflammatory agent is relatively small. It is impossible to determine beforehand which patients possess only irreversible joint swelling. The results obtained from comparative studies of groups are diluted by the presence of patients without reversible inflammatory swelling. Although trends may be demonstrated, this factor may prevent the attainment of statistically significant differences.

It is probable that selection is not of importance in trials such as those reported in this study, in which each patient serves as his own control. The only trial in which a relatively large number were excluded from analysis was the comparison of low dosage of salicylate with placebo, trial 4. The mean age of the 15 patients included was 42 years: 12 were females and three males. The mean age of those excluded was 46 years : six were females and three males.

The trial consisted of two periods, each of seven days. This short duration was chosen to minimize variation due to the natural history of the disease. It was thought preferable to keep the administration of prednisone to a minimum. It was noted in patients studied in hospital that changes in joint size after the administration of rapidly acting anti-inflammatory agents are rapid, often occurring maximally within 48 hours (Hart and Boardman, 1963). In the presence of a limited amount of inflammatory swelling it is possible that any factor promoting improvement is associated with reduction of joint size, provided the period of administration is long enough. In these circumstances differences may be demonstrated more clearly by comparing the rate of change during a limited period.

The use of a cross-over trial may be criticized. because subiects starting on an active agent may have less chance of responding in the second trial period. Rebound exacerbation after corticosteroid withdrawal may also affect the findings. The results obtained suggest that this is relatively unimportant under these defined conditions. It is unlikely that the order of administration affected the objective measurement of joint size in the three trials involving placebo. In the comparison of high and low dosage of salicylate analysis revealed no pattern of response that might depend on the order of the drugs. In the first week on high dosage improvement of joint size occurred in five out of seven, and in the second week in 7 out of 11 . In the first week on low dosage 1 out of 11 improved, and in the second week 1 out of 7 . The mean improvement in joint size on high dosage of salicylate in the first period was $+5 \mathrm{~mm}$.; in the second period it was $+4 \mathrm{~mm}$.

Clinically, joint size may consist of irreversible swelling and potentially reversible disease, the latter including inflammatory oedema, stasis oedema, and vascular dilatation. Irreversible swelling is excluded by the sequential method of analysis. Vascular dilatation is relatively unimportant, as the mean increase of joint size in a group of eight patients with rheumatoid arthritis and in three healthy control subiects after exercise and heat was about $1 \mathrm{~mm}$. for the 10 joints of the hands. The changes in joint size were not the result of the elimination of stasis oedema after increased activity from satisfactory analgesia, because paracetamol alone was not associated with alteration of joint size. The apparent diurnal variation of joint size may be related to immobility during the night. In a further attempt to avoid this factor patients were assessed in the afternoon. The changes of joint size are therefore most likely to reflect alteration in inflammatory swelling.

Side-effects were no problem. It is of interest that drowsiness or dyspepsia occurred in over $50 \%$ of those receiving paracetamol. Tinnitus was the main complaint on high dosage of salicylate.

These findings confirm the importance of administering high dosage of salicylates in rheumatoid arthritis, irrespective of symptoms and their severity, if the aim of treatment is the promotion of nonspecific anti-inflammatory action. Relief of pain by low dosage of salicylate appears to be strictly limited and anti-inflammatory activity nonexistent under the conditions defined.

The measurement of joint swelling is accurate under controlled conditions. The response is so small that factors such as observer variation and differences in the time of the day may introduce error greater than the actual response.

In comparative drug trials the unrestricted use of salicylates, to be taken by the patient whenever necessary for supplementary analgesia, affects the results obtained, particularly at high dosage. If the number of tablets ingested by each group is different there may be selective alteration of the criteria of inflammatory activity. The number of tablets ingested in each period is therefore not a satisfactory index of progress. Low dosage of salicylate should be avoided, as it is probable that in combination it affects the response to other agents of indices such as joint size. Jick, Pinals, Ullian, Slone, and Muench (1965), using sequential analysis, showed that the addition of aspirin in small doses ( $1.5 \mathrm{~g}$. daily) increased the therapeutic efficiency of dexamethasone $0.75 \mathrm{mg}$. daily to the level of $1.5 \mathrm{mg}$. daily.

It is suggested that for the assessment of anti-inflammatory effect of rapid onset in patients measurement of joint size under controlled conditions may be used, the results being interpreted by sequential analysis.

\section{Conclusions}

Joint size may be measured serially with reasonable accuracy under controlled conditions for the clinical assessment of inflammation in rheumatoid arthritis. 
The patient's preference and grip strength indicate improvement on drugs which are anti-inflammatory or analgesic agents ; under defined conditions joint size is an index of specific antiinflammatory effect.

The reduction of joint size by prednisone, an antiinflammatory agent, is contrasted with the absence of change on paracetamol.

High dosage of salicylate, unlike low dosage, is associated with reduction in joint size.

There is no detectable difference between low dosage of salicylate and placebo in their effect on joint size.

It is suggested that high dosage of salicylate, unlike low dosage, has a clinically measurable anti-inflammatory effect. If suppression of inflammation is desirable in rheumatoid arthritis high dosage of salicylate is indicated independently of symptoms.

Unlimited use of high dosage of salicylate in trials may affect the findings. Low dosage modifies the response if given in combination. Salicylate intake should be eliminated from comparative trials of anti-inflammatory agents.

It is suggested that the plan of this trial provides a simple method for the clinical assessment of the anti-inflammatory properties of a rapidly acting drug.
We wish to thank Dr. Peter Fowler for providing the millimetre gauge for the measurement of joint size; Dr. E. Lewis-Faning for statistical advice; and Mr. J. A. Baker, chief pharmacist at the hospital, for organizing the provision of tablets suitable for the trials. Our thanks are also due to many members of the unit and to students for assisting in the assessment of error, in particular for the work illustrated in Fig. 2.

The above work formed the basis of a paper read to the Heberden Society in June 1967.

\section{REFERENCES}

Adams, S. S., and Cobb, R. (1963). In Salicylates, An International Symposium, edited by A. St. J. Dixon, B. K. Martin, M. J. H. Symposium, edited by A. St. J. Dixon, B. K
Smith, and P. H. N. Wood, p. 127. London.

American Rheumatism Association (1959). Ann rheum. Dis., 18, 49.

Bywaters, E. G. L. (1963). In Salicylates, An International Symposium edited by A. St. J. Dixon, B. K. Martin, M. J. H. Smith, and P. H. N. Wood, p. 154 . London.

Fremont-Smith, K., and Bayles, T. B. (1965). 7. Amer. med. Ass., $192,1133$.

Hart, F. D., and Boardman, P. L. (1963). Brit. med. F., Q, 965.

- and Clark, C. J. M. (1951). Lancet, 1, 775.

Jick, H., Pinals, R. S., Ullian, R., Slone, D., and Muench, H. (1965) Ibid., 2, 1203 .

Smyth, C. J., and Clark, G. M. (1957). f. chron. Dis., 5, 734.

Vaughn, P. P., Howell, D. S., and Kiem, I. M. (1959). Arthr. and Rheum., 2, 212

Wright, V. (1959). Clin. Sci., 18, 17.

\title{
Glyceryl Trinitrate in Angina Pectoris: Tablet or Aerosol ?
}

\author{
G. SANDLER, ${ }^{*}$ M.D., M.R.C.P. ; G. A. CLAYTON, $\dagger$ M.B., CH.B
}

Brit. med. F., 1967, 4, 268-270

Though many drugs have been used in the symptomatic treatment of angina pectoris almost the only one which has been consistently shown to be of benefit in relieving the myocardial ischaemia is glyceryl trinitrate. In a previous study it was shown to be of considerable value when used prophylactically, but no better than a placebo when given after an attack of anginal pain had developed (Sandler et al., 1963). It has been suggested that this lack of effect of gylceryl trinitrate in relieving established anginal pain might be due to the time it took to dissolve in the mouth (Evans, 1963), so that by then the angina had improved spontaneously. In relation to this possibility the development of an aerosol preparation of glyceryl trinitrate seemed of considerable interest, since such a preparation would be rapidly absorbed in the lungs with more rapid arrival in the myocardium. Preliminary observations (G. G. Gensini, unpublished; A. Black, unpublished) suggested that glyceryl trinitrate aerosol was of value in treating angina, and it was therefore decided to undertake an objective evaluation of the aerosol preparation (Cardamist) in treating angina pectoris and to compare it with the standard tablet trinitrate preparation.

\section{Patients and Methods}

Twenty-three patients with well-authenticated and typical attacks of angina pectoris were studied. These subjects, aged from 39 to 69 years, comprised 20 men and 3 women. In all of them the angina had settled down to a stable pattern, and the duration ranged from 3 to 72 months, with attacks occurring from 3 to 40 times weekly. The presumptive cause of the angina was coronary artery disease in all 23 , but only four had evidence of previous myocardial infarction.

- Consultant Physician, St. Helen Hospital, Barnsley.

+ Medical Registrar, St. Helen Hospital, Barnsley.
All the patients were admitted to hospital for a period of six days for the trial, and a daily exercise tolerance was carried out in each of them. The type of test has been described in detail in an earlier publication (Sandler, 1961), and is based on a modification of the Master two-step test, the patient exercising over two steps, each 9 in. $(23 \mathrm{~cm}$.) high, until he develops angina or completes 100 circuits over the steps; the test is stopped immediately if significant ischaemic change appears in the electrocardiogram during exercise. In addition to conventional electrocardiography, recording chest lead V5 before and after exercise, the patient is monitored continuously during exercise by means of a radiocardiograph. By this means ischaemic change can be detected immediately, even if unaccompanied by anginal pain, and the exercise terminated (Sandler, 1967). Depression of the ST segment of plane or sagging contour lasting at least 0.08 second, in either the radiocardiogram or the V5, was taken as evidence of myocardial ischaemia (Lloyd-Thomas, 1961 ; Master and Rosenfeld, 1961 : Bellet et al., 1962). Junctional depression, when it occurred, was accepted as significant of ischaemia only when the QX/QT ratio exceeded 50\% (Master and Rosenfeld, 1961).

The preparations studied were glyceryl trinitrate aerosol delivering $0.13 \mathrm{mg}$. of the drug per inhalation-the dose investigated being $0.26 \mathrm{mg}$. (two inhalations)-an identical placebo aerosol, and standard tablets of $0.5 \mathrm{mg}$. of glyceryl trinitrate. Study of the aerosol preparations was made on a double-blind basis, and the distribution of active and placebo preparations in each pair of aercsols was randomized. It was not thought necessary to include a placebo tablet with the glyceryl trinitrate tablet, since a previous double-blind study had confirmed the effectiveness of the latter in this type of trial (Sandler et al., 1963). Each of the three preparationsglyceryl trinitrate aerosol, placebo aerosol, and glyceryl trinitrate tablet-was assessed when given both before exercise was 